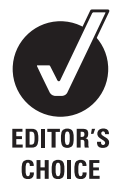

${ }^{1}$ Department of Ophthalmology, Karolinska Institutet, St Eriks Eye Hospital, Stockholm,

Sweden

${ }^{2}$ Ophthalmology, Department of Neuroscience, Uppsala University, Uppsala, Sweden

\section{Correspondence to}

Dr Anders Kvanta, Department of Ophthalmology, St Eriks Eye Hospital, Polhemsgatan 50, SE-11282 Stockholm, Sweden anders.kvanta@sankterik.se

Accepted 7 December 2011 Published Online First

12 January 2012

\title{
Combination therapy with low-dose transpupillary thermotherapy and intravitreal ranibizumab for neovascular age-related macular degeneration: a 24-month prospective randomised clinical study
}

\author{
Anne-Catherine Söderberg, ${ }^{1}$ Peep V Algvere, ${ }^{1}$ Jürg C Hengstler, ${ }^{1}$ Pär Söderberg, ${ }^{2}$ \\ Stefan Seregard, ${ }^{1}$ Anders Kvanta ${ }^{1}$
}

\section{ABSTRACT}

Aim To compare the effect of combined low-dose transpupillary thermotherapy (TTT) and intravitreal ranibizumab with sham $\Pi T$ and intravitreal ranibizumab in patients with neovascular age-related macular degeneration (AMD).

Methods A 24-month, double-masked, randomised, active-controlled clinical trial. 100 patients with primary neovascular AMD were randomly assigned (1:1) to receive intravitreal ranibizumab and sham TTT or intravitreal ranibizumab and low-dose TTT. After an initial loading phase of ranibizumab patients were assigned to receive quarterly low-dose $T T T(136 \mathrm{~mW} / \mathrm{mm})$ or sham TTT for 24 months. Retreatment with ranibizumab was allowed in both treatment groups using a variable dosing regimen. The primary endpoint was the number of intravitreal injections with ranibizumab. Secondary endpoints included change in best corrected visual acuity (BCVA), central retinal thickness (CRT) and lesion area.

Results In the per protocol (PP) population (78 patients) the mean number of ranibizumab injections was 8.0 in the sham TTT group versus 6.3 in the TTT group $(p<0.05)$. The mean number of injections between $0-12$ months and $13-24$ months was 4.8 versus 4.6 $(p>0.05)$ and 3.2 versus $1.7(p<0.01)$ in the sham TTT and TTT groups, respectively. There was no statistically significant difference in BCVA (+4.0 vs +0.9 ETDRS letters), CRT ( $-49.9 \%$ vs $-36.4 \%$ ) or lesion area $(-0.3 \%$ vs $-10.6 \%)$ between the treatment groups at the final examination. The results of the intent-to-treat population (92 patients) were similar to the PP population.

Conclusions Treatment with low-dose TTT significantly reduced the number or intravitreal injections of ranibizumab over 24 months. The results suggest that low-dose TTT can serve as an adjuvant in combination with intravitreal ranibizumab for neovascular AMD.

Clinical trial registration number The trial is registered at http://clinicaltrails.gov (no NCT00599222).

Neovascular age-related macular degeneration (AMD) characterised by choroidal neovascularisation is a primary cause of legal blindness in the western world. Without treatment, prognosis is poor, resulting in a rapid and progressive loss of visual acuity and contrast sensitivity. The introduction of drugs that target vascular endothelial growth factor, a major factor in the pathogenesis of choroidal neovascularisation, is a breakthrough in the management of neovascular AMD. ${ }^{1} 2$
Randomised trials have shown that intravitreal ranibizumab (Lucentis) on a monthly dosing regimen stabilises visual acuity in over $90 \%$ and improves it in over $30 \%$ of cases. ${ }^{1}{ }^{2}$ Monthly treatment is, however, more costly and logistically demanding, and increases the risk of complications (including endophthalmitis). For these reasons, ranibizumab is often provided using a variable dosing regimen after an initial loading phase of 3-monthly injections. However, there seems to be a clinical superiority with more frequent dosing. ${ }^{3-5}$ In this context, a combination therapy that would reduce the number of intravitreal ranibizumab injections while maintaining visual outcome would be an attractive alternative.

Transpupillary thermotherapy (TTT) is a technique to induce vascular occlusion by delivering radiation near infrared $(810 \mathrm{~nm})$ to the target tissue through the pupil. TTT causes a small temperature elevation $\left(8-10^{\circ} \mathrm{C}\right)$ of choroidal lesions with subsequent intravascular thrombosis. ${ }^{6-9}$ Initially, there was concern regarding the clinical safety of TTT when using a dose of $248 \mathrm{~mW} / \mathrm{mm}$. However, we have recently shown that a low (sub-threshold) dose of TTT $(136 \mathrm{~mW} / \mathrm{mm})$ can be applied to patients with neovascular AMD without apparent damage to the surrounding neurosensory retina. ${ }^{10}$ In a randomised study that compared low-dose TTT and photodynamic therapy (PDT) both treatments stabilised visual acuity in over $70 \%$ of patients with occult choroidal neovascularisation. ${ }^{10}$ Experimental studies in murine models have shown that both PDT and TTT cause prolonged vascular occlusion to the target tissue, a distinctly different mode of action compared with anti-vascular endothelial growth factor drugs. ${ }^{11} 12$ This in turn suggests a potential adjuvant effect of these laserbased therapies when combined with intravitreal ranibizumab. The concept has been studied in smaller trials and is currently being investigated in the MONT BLANC and DENALI multicentre studies. ${ }^{13-16}$

The primary objective of this study was to investigate whether quarterly treatment with lowdose TTT can reduce the number of re-injections of intravitreal ranibizumab using a variable dosing regimen in patients with neovascular AMD.

\section{METHODS}

This investigation was approved by the Regional Ethical Review Board in Stockholm and by the 
Swedish Medical Products Agency (EU-no 2007-005462-12). The trial is registered at http://clinicaltrails.gov (no NCT00599222). All patients included gave their informed consent to participate.

\section{Study design and patients}

One hundred patients (100 eyes) were enrolled and randomly assigned (in a 1:1 ratio) to either low-dose TTT and ranibizumab or sham TTT and ranibizumab. For each consecutive patient a sealed envelope containing assignment to either sham TTT or low-dose TTTwas drawn from a pool of 100 envelopes by study personnel not involved in the follow-up. Six randomly assigned patients did not receive any study treatment (TTT or sham TTT) and were therefore excluded from the study (see table 1 for details). Two randomly assigned patients were, on subsequent assessments, found to have branch retinal vein occlusion and were excluded. The remaining 92 patients comprised the intent-to-treat (ITT) population in which the last observation carried forward (LOCF) method was used for missing data for all efficacy parameters. Analysis was performed on the ITT population and on the per protocol (PP) population (78 patients) defined as study subjects completing the 24-month follow-up without major protocol deviations.

To be eligible for the study the patients had to meet the following inclusion criteria: (1) age 50 years or older; (2) choroidal neovascularisation (predominantly classic, minimally classic or occult) under the geometric centre of the fovea; (3) best corrected visual acuity (BCVA) corresponding to a Snellen equivalent of 20/200 to 20/40. Exclusion criteria included: (1) patients with BCVA corresponding to a Snellen equivalent below 20/200; (2) subretinal fibrosis or atrophy; (3) indocyanine angiography verified polypoidal choroidal vasculopathy; (4) previous treatment with anti-angiogenic drugs, PDT, TTT or vitrectomy; (5) cardiovascular or cerebrovascular events within 12 months.

Each patient received a standard loading dose of monthly intravitreal ranibizumab for 3 months. Additional re-injections were given if any of the following criteria were fulfilled: (1) BCVA loss of at least five ETDRS letters; (2) increase in optical coherence tomography (OCT) central retinal thickness (CRT) of

Table 1 Summary of the study patient disposition

\begin{tabular}{llll}
\hline Patient enrolment & Reason for discontinuation & Sham TTT & TTT \\
\hline Enrolled & & 50 & 50 \\
Randomised & 50 & 50
\end{tabular}

Excluded from study

Discontinued treatment

Erroneous diagnosis on review

2

before first TTT

Received randomised

treatment

Discontinued treatment

before month 24

$\begin{array}{lrr}\text { Patient's decision } & 1 & 1 \\ \text { Adverse event before first TTT* } & 3 & 1 \\ & 44 & 48 \\ & 4 & 11\end{array}$

Adverse event $\dagger \quad 114$

Patient's decision

Unsatisfactory therapeutic effort 3

Protocol deviation

Remained on treatment at month 24
*Sham transpupillary thermotherapy (TTT) (one massive submacular haemorrhage, one retinal pigment epithelium rip, one severe vision loss ( $>30$ letters)), TTT (one severe vision loss (>30 letters)).

†Sham TTT (one death), TTT (three deaths, one stroke). at least $100 \mu \mathrm{m}$; (3) new classic choroidal neovascularisation; (4) new submacular haemorrhage; (5) persistent intra or subretinal fluid. All criteria were based on a comparison with the previous clinical examination or the last fluorescein angiography. Low-dose TTT or sham TTT (active control) was applied on a fixed regimen every 3 months starting 1 month after the last loading injection (ie, total of six TTT treatments over 24 months).

\section{Treatment}

The intraocular pressure was checked and biomicroscopy of the anterior and posterior segments of the eye carried out before the intravitreal injection. The preoperative measures included dilatation of the pupil, prophylactic administration of Fucithalmic ointment, periocular skin cleaning with chlorohexidine solution $(5.0 \%)$ and irrigation of the conjunctiva with chlorohexidine solution $(0.5 \%)$. The intravitreal injection was performed under an operating microscope in a room dedicated for intraocular surgery under aseptic conditions. Using a 30-gauge needle on a $1 \mathrm{ml}$ syringe, $0.5 \mathrm{mg}(0.05 \mathrm{ml})$ of a commercially available solution of Lucentis (Novartis Ophthalmics, Basel, Switzerland) was injected $3.5-4.0 \mathrm{~mm}$ from the limbus under microscopic inspection.

TTT was applied as previously described. ${ }^{10}$ Briefly, an infrared diode laser at $810 \mathrm{~nm}$ (Iridex Corporation, Mountain View, California, USA) delivering a single spot for $60 \mathrm{~s}$ via a slit-lamp adapter was used. The Mainster wide field lens was used with a standard $3 \mathrm{~mm}$ spot in the laser slit lamp, which produced a spot size of $4.4 \mathrm{~mm}$ in diameter on the retina. The laser was selected to deliver $600 \mathrm{~mW}$, corresponding to a power of $136 \mathrm{~mW}$ for a circular spot of $1 \mathrm{~mm}$ diameter. As the temperature increases linearly with increased laser spot diameter, treatment power is also proportional to treatment spot diameter. Therefore, a $1.5 \mathrm{~mm}$ diameter circular lesion requires half the laser power of a $3.0 \mathrm{~mm}$ lesion. Sham TTT was performed as above without the laser being turned on.

\section{Assessments}

Clinical assessments at baseline and at the monthly visits included BCVA (logarithmic ETDRS chart at $4 \mathrm{~m}$ ), slit-lamp biomicroscopy of the anterior and posterior segments, intraocular pressure measurement and OCT (Stratus OCT; ZeissHumphrey (Carl Zeiss AB, Stockholm, Sweden), Cirrus OCT; Zeiss-Humphrey). Due to the fact that two different OCT machines were used in this trial (with different segmentation algorithms) CRTwas manually measured at the presumed centre from the top of the internal limiting membrane reflectivity to the top of the reflectivity of the retinal pigment epithelium. These measurements were performed by a single examiner. Fluorescein and indocyanine angiography were performed at baseline, and at 12 and 24 months. Experienced ophthalmologists masked to the treatment of the patient evaluated all examinations of angiograms and OCT.

\section{Power calculation and statististical methods}

The primary endpoint was the number of re-injections of ranibizumab over the 24-month study period. Secondary endpoints were change in BCVA, the proportion of patients with stabilised (loss of $<15$ letters) or improved (gain of $\geq 15$ letters) BCVA, lesion area and CRT. Primary and secondary endpoints were measured on both the PP and ITT (LOCF) populations. Data obtained from patients who withdrew from the study after randomisation but before receiving their first TTT treatment were excluded (see table 1). Based on our clinical observations on 
the expected re-injection rate we estimated that the sham TTT group would receive an average of eight plus or minus four injections (five re-injections) versus six plus or minus three injections for the low-dose TTT group over 24 months. With a statistical power of $80 \%$ and the level of statistical significance set at $\mathrm{p}<0.05$ we calculated that a minimum of 50 patients would be required per group (MedCalc Software, Mariakerke, Belgium). For statistical analyses the Student's t test was used for comparisons of independent means, the $\chi^{2}$ test for comparisons of two proportions and the analysis of variance test for repeated measurements of changes in BCVA. Statistical significance was set at $\mathrm{p}<0.05$.

\section{RESULTS}

One hundred patients with neovascular AMD were enrolled and randomly assigned to receive either low-dose TTT or sham TTT. Eight patients were excluded (table 1) and the remaining 92 patients (TTT, $n=48$; sham TTT, $n=44$ ) received active or sham TTT. These patients comprised the ITT (LOCF) population. The PP population comprised 78 patients (TTT, $n=38$; sham TTT, $\mathrm{n}=40$ ). At baseline, there was no statistical difference in age, gender, BCVA, CRT, lesion size or type between the treatment groups (table 2).

The mean change in BCVA over time between the treatment groups is shown in figure 1. A rapid increase in BCVA was observed after the loading phase in both the PP (figure 1A) and ITT (figure 1B) populations. After the loading phase, BCVA in the PP population had increased by 8.8 and 5.3 letters in the two treatment groups, respectively. This non-statistically significant difference between the groups was noted before TTT was initiated and was maintained throughout the study. During the maintenance (sham TTT/TTT) phase BCVA slowly decreased, reaching near baseline levels after 24 months. The final change in BCVA (mean \pm SE) was $+4.0 \pm 1.8$ and $+1.0 \pm 2.8$ for the sham TTT and TTT groups, respectively $(\mathrm{p}>0.05)$. The proportion of patients with stabilised visual acuity (lost $<15$ letters) was $90.0 \%$ and $79.9 \%$ in the sham TTT and TTT groups, respectively $(\mathrm{p}>0.05)$. The corresponding proportion of patients who had a visual acuity gain of 15 or more letters was $7.5 \%$ in the sham group and $18.4 \%$ in the TTT group ( $p>0.05$ ). The visual acuity

Table 2 Demographics and baseline charatersistics of the ITT population

\begin{tabular}{|c|c|c|}
\hline Charateristic & Sham TTT $(n=44)$ & $\mathrm{TTT}(\mathrm{n}=48)$ \\
\hline \multicolumn{3}{|l|}{ Gender, n (\%) } \\
\hline Women & $18(40.9)$ & $28(57.1)$ \\
\hline Men & $26(59.1)$ & $21(42.9)$ \\
\hline \multicolumn{3}{|l|}{ Age (years) } \\
\hline Mean (SD) & $78.4(7.9)$ & $79.5(8.0)$ \\
\hline \multicolumn{3}{|l|}{ BCVA (letters) } \\
\hline Mean (SD) & $57.3(13.9)$ & $58.6(12.9)$ \\
\hline \multicolumn{3}{|c|}{ Choroidal neovascularisation classification, $\mathrm{n}(\%)$} \\
\hline Predominantly classic & $16(36.4)$ & $10(22.7)$ \\
\hline Minimally classic & $5(11.4)$ & $10(20.8)$ \\
\hline Occult (no classic) & $14(31.8)$ & $16(33.3)$ \\
\hline RAP & $9(20.4)$ & $12(25.0)$ \\
\hline \multicolumn{3}{|l|}{ Retinal thickness $(\mu \mathrm{m})$} \\
\hline Mean (SD) & 367 (149) & $328(133)$ \\
\hline \multicolumn{3}{|l|}{ Mean lesion area $\left(\mathrm{mm}^{2}\right)$} \\
\hline Mean (SD) & $2.8(1.2)$ & $3.3(1.2)$ \\
\hline
\end{tabular}
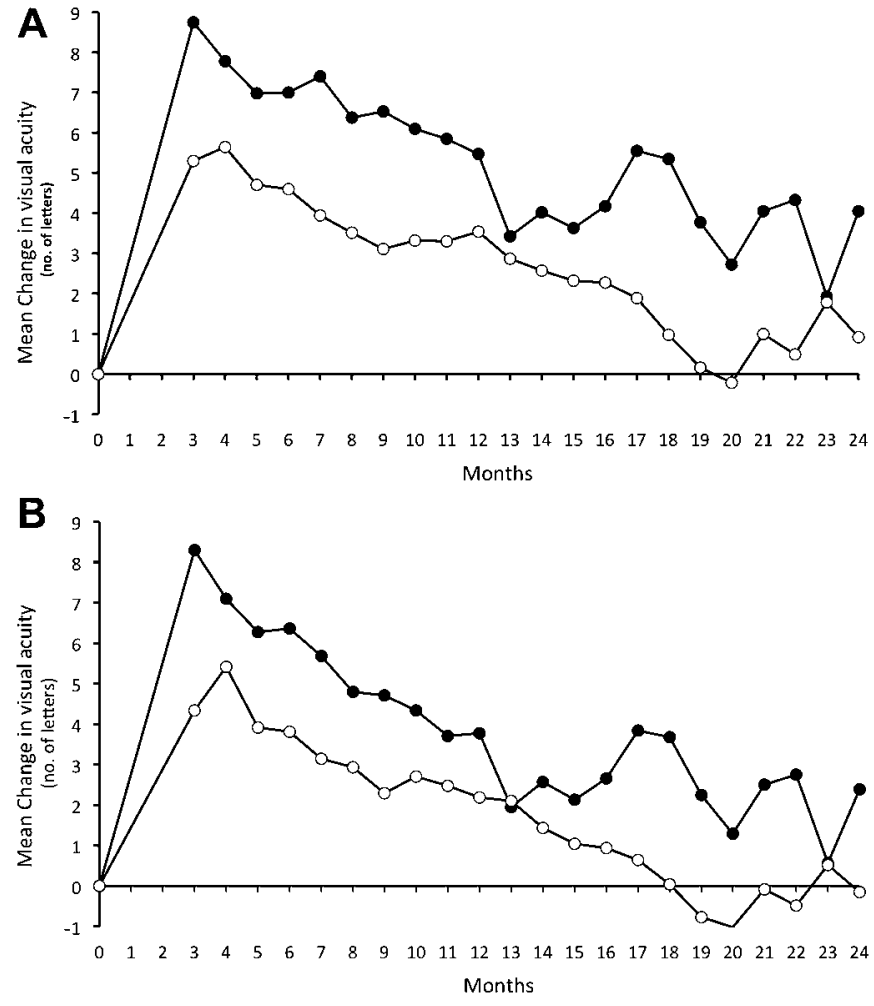

Figure 1 Best corrected visual acuity in the per protocol $(A)$ and intentto-treat (B) population treated with sham transpupillary thermotherapy (TTT) and low-dose TTT combined with intravitreal ranibizumab. Sham TTT, closed circles; low-dose TTT, open circles.

results of the ITT population were consistent with the PP population (not shown).

The primary endpoint of this study was the number of ranibizumab injections over 24 months. Data for the PP and ITT populations are shown in figure 2 . The mean number of injections ( $( \pm \mathrm{SE})$ including the three loading doses) over the full 24month study period in the PP population was significantly lower in the TTT group $(6.3 \pm 2.8$ injections) than in the sham TTT group ( $8.0 \pm 3.3$ injections) $(p<0.05)$. There was no statistical difference during the initial 12-month study period but during the final 12 months the number of re-injections was significantly reduced in the TTT group $(\mathrm{p}<0.01)$ (figure $2 \mathrm{~A})$. The proportion of patients receiving three or more re-injections during this period was $23.7 \%$ for the low-dose TTT group and $62.5 \%$ for the sham TTT group $(p<0.01)$. This translated into a reduction by $45 \%$ in the TTT group (1.7 injections) versus sham TTT (3.2 injections) during the second study year. The re-injection results of the ITT population (figure $2 \mathrm{~B}$ ) were consistent with the PP population. Subgroup analysis revealed a marked decrease in re-injections in the low-dose TTT group for all lesion types except for retinal angiomatous proliferation (RAP) (table 3).

CRT in the PP population was reduced in both the sham TTT $(375-206 \mu \mathrm{m}, \mathrm{p}<0.05)$ and TTT groups $(319-220 \mu \mathrm{m}, \mathrm{p}<0.05)$. The ITT population showed a similar decrease between the groups.

The results of secondary outcome measures are summarised in table 4 . The mean lesion size was stable in the sham TTT group, whereas there was a small reduction in lesion size in the TTT group ( $p>0.05$ ). Persistent leakage was higher in TTT-treated patients (23.1\% vs $34.8 \%)$. On the other hand, the TTT group showed less fibrosis on the final angiograms (34.8\% vs $51.3 \%$ ). 

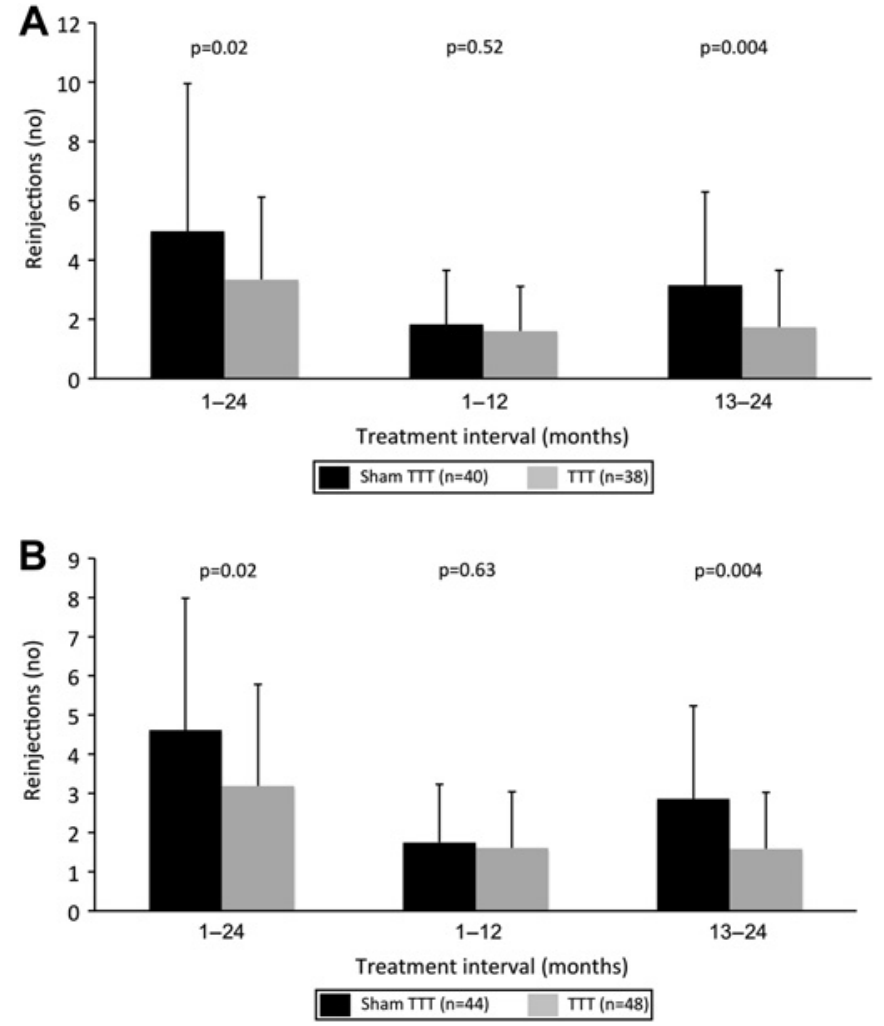

Figure 2 Re-injections in the per protocol $(A)$ and intent-to-treat $(B)$ population treated with sham transpupillary thermotherapy (TTT) and low-dose TTT combined with intravitreal ranibizumab.

None of these differences between treatment groups were statistically significant ( $\mathrm{p}>0.05)$.

\section{DISCUSSION}

In this prospective, randomised study on patients with neovascular $\mathrm{AMD}$ we detected a statistically significant reduction in the number of intravitreal ranibuzimab re-injections when combining the treatment with quarterly low-dose TTT. There was consistency between the PP and ITT populations, and the results provide support for combination treatment in neovascular AMD.

One combination approach currently being investigated is PDT and intravitreal ranibizumab. Available results from smaller studies have not confirmed an adjuvant effect of PDT. In a recent study, 18 patients were randomly assigned to either standard or sham PDT combined with a flexible ranibizumab dosing regimen. After the 1-year follow-up period, BCVA (+2.2 vs +4.4
Table 3 Re-injection by lesion type

\begin{tabular}{llll}
\hline Lesion type & Sham TTT & TTT & \% Decrease \\
\hline Predominantly classic & $3.5(3.0)$ & $2.0(2.6)$ & 43 \\
Minimally classic & $5.4(3.8)$ & $2.5(1.6)$ & 54 \\
Occult (no classic) & $4.9(3.5)$ & $3.4(2.7)$ & 31 \\
RAP & $4.6(3.1)$ & $4.4(2.5)$ & 4 \\
\hline
\end{tabular}

Mean (SD) number of re-injections by angiographic lesion subtype over the 24-month study period.

RAP, retinal angiomatous proliferation; TTT, transpupillary thermotherapy.

ETDRS letters) or re-injection rate (1.3 vs 1.3 ) was similar. ${ }^{16}$ More detailed information on the potential efficacy of adjuvant PDT will be available when results of the ongoing multicentre MONT BLANC and DENALI studies are published.

Instead, we chose to use low-dose TTT as an adjuvant. There are several reasons for this decision. First, in an experimental study we showed that TTT reduces choroidal neovascularisation formation even at doses that spare the overlying neurosensory retina. ${ }^{11}$ At the ultrastructural level, TTT caused permanent vascular occlusion and involution of the choroidal neovascularisation lesions. Second, in a randomised study in patients with occult choroidal neovascularisation we showed that lowdose TTT and standard PDT have a similar efficacy and safety profile as measured by the stabilisation of BCVA and the preservation of patient-reported quality of life. ${ }^{10} 17$ Third, the cost of TTT is negligible and the technique is easy to manage compared with PDT.

In the pivotal MARINA and ANCHOR trials, an increased BCVA that remained stable during the 2-year study period was achieved with the monthly injection regimen. ${ }^{1} 2$ In practice, most clinicians use a variable dosing regimen sometimes at the expense of a lower BCVA outcome than in the pivotal studies. Indeed, most studies, using a variable dosing regimen, have not been able to maintain the initial improvement in BVCA seen after the loading phase. ${ }^{3}{ }^{4}$ However, in the recently published Comparative AMD Treament Trial (CATT) study very good BCVA results over 12 months were achieved when using flexible dosing and strict re-injection criteria. ${ }^{5}$ In the present study, we used a dosing regimen similar to the PrONTO trial in which ranibizumab re-injections are given according to specified criteria. ${ }^{18}$ As expected, we also found that after the initial increase in BCVA following the loading phase BCVA gradually declined over the 2-year study period. The overall stabilisation in visual acuity was similar to previous studies. ${ }^{3} 419$

In the present study, stabilisation of visual acuity was achieved with 4.8 and 3.2 injections during years 1 and 2, respectively. This is lower than reported in the SUSTAIN and PrONTO studies, in which the mean number of injections during the first study year was 5.6 over 12 months $^{18} 19$ and 9.9

Table 4 Secondary outcome measures in the ITT/LOCF population

\begin{tabular}{|c|c|c|c|c|c|c|c|c|c|c|}
\hline & Sham TTT baseline & Sham TTT 24 months & Difference $(\%)$ & p Value & $\bar{n}$ & TIT baseline & TIT 24 months & Difference (\%) & p Value & $\bar{n}$ \\
\hline \multicolumn{11}{|l|}{ Area $\left(\mathrm{mm}^{2}\right)$} \\
\hline Mean (SD) & $2.86(1.19)$ & $2.85(1.22)$ & $-0.30 \%$ & $>0.05$ & 39 & $3.31(1.23)$ & $2.96(1.40)$ & $-10.60 \%$ & $>0.05$ & 45 \\
\hline \multicolumn{11}{|l|}{ Leakage } \\
\hline \multicolumn{11}{|l|}{ Fibrosis } \\
\hline $\mathrm{n}(\%)$ & & $20(51.3)$ & & & 39 & & 16 (34.8) & & & 45 \\
\hline \multicolumn{11}{|l|}{ CRT $(\mu \mathrm{m})$} \\
\hline
\end{tabular}

Angiographic characteristics (choroidal neovascularisation lesion area, leakage and fibrosis) and central retinal thickness (CRT) in the sham transpupillary thermotherapy (TTT) and low-dose TTT-treated groups at baseline and 24 months.

ITT, intent-to-treat; LOCF, last observation carried forward. 
over 24 months, ${ }^{18}$ but higher than in other studies using similar flexible dosing regimens. ${ }^{16}$

The main finding of the current study was the reduction in the re-injection rate in the low-dose TTT group compared with the sham TTT group during the second study year. Previous experimental and clinical data suggest that repeated low-dose TTT causes vascular thrombosis and gradual involution of choroidal neovascularisation lesions. This would in turn decrease vascular growth and leakage, limiting the need for ranibizumab re-injections. Therefore, repeated TTT in this set of patients may create a localised fibrosis of choroidal neovascularisation. It is of importance that such an effect may be achieved with minimal collateral damage to the overlying neurosensory retina. We did observe that more patients (not statistically significant) in the TTT group lost more than 15 letters after 24 months and that may be a reason for some safety concerns. On the other hand, more patients also gained 15 or more letters in the TTT group compared with the sham TTT group. Notably, the BCVA and CRT in the sham TTT and TTT groups were comparable at 24 months. Also, an analysis of the final angiograms did not suggest a profibrotic effect of TTT (not shown). In fact, the overall number of fibrotic lesions was lower in the TTT group, whereas the number of lesions with persistent leakage was higher. Together, these observations suggest that TTT-induced scarring did not cause the difference in the ranibizumab reinjections rate. Rather, it is more likely that vascular occlusion leading to involution of lesions reduced the need for ranibizumab re-injection.

Overall, lesion subgroup analysis revealed no apparent differences in the re-injection rate. However, an interesting observation was the lack of TTT-induced reduction in re-injections in patients with RAP lesions. Although the subgroups were small one can speculate that TTT is less efficient in RAP in which the pathology resides within the neurosensory retina rather than in the subretinal and choroidal space.

With the introduction of intravitreal ranibizumab we now have a powerful treatment for neovascular AMD. However, this success comes at a high cost, burdensome logistics and the fact that a long-term treatment effect is not permanent. The results of the present study suggest that low-dose TTT can, over time, decrease the number of ranibizumab re-injections. Therefore, in the search for treatments that can serve as adjuvants to intravitreal ranibizumab, low-dose TTT is an attractive candidate.

Competing interests None.

Ethics approval This investigation was approved by the Regional Ethical Review Board in Stockholm and by the Swedish Medical Products Agency (EU-nr 2007-005462-12).

Contributors ACS performed the bulk of the clinical assessment, contributed to study design and data evaluation. PVA contributed to study design and assisted with manuscript preparation. JCH assisted in clinical assessment. PS contributed to the statistical evaluation and data management. SS contributed to study design, data evaluation and manuscript preparation. AK contributed to study design, statistical analysis, data management, data evaluation and manuscript preparation.

Provenance and peer review Not commissioned; externally peer reviewed.

\section{REFERENCES}

1. Rosenfeld PJ, Brown DM, Heier JS, et al. Ranibizumab for neovascular age-related macular degeneration. N Engl J Med 2006:355:1419-31.

2. Brown DM, Kaiser PK, Michels $M$, et al. Ranibizumab versus verteporfin for neovascular age-related macular degeneration. $N$ Engl J Med 2006;355:1432-44.

3. Schmidt-Erfurth U, Eldem B, Guymer R, et al. Efficacy and safety of monthly versus quarterly ranibizumab treatment in neovascular age-related macular degeneration: the EXCITE Study. Ophthalmology 2011;118:831-9.

4. Regillo CD, Brown DM, Abraham P, et al. Randomized, double-masked, sham-controlled trial of ranibizumab for neovascular age-related macular degeneration: PIER Study year 1. Am J Ophthalmol 2008;145:239-48.

5. Martin DF, Maguire MG, Ying GS, et al; CATT Research group. Ranibizumab and bevacizumab for neovascular age-related macular degeneration. N Engl J Med 2011;364:1897-908.

6. Algvere PV, Libert C, Lindgärde G, et al. Transpupillary thermotherapy of predominantly occult choroidal neovascularization in age-related macular degeneration with 12 months follow-up. Acta Ophthalmol Scand 2003;81:110-17.

7. Lanzetta P, Michieletto P, Pirracchio A, et al. Early vascular changes induced by transpupillary thermotherapy of choroidal neovascularisation. Ophthalmology 2002;109:1098-104.

8. Mainster MA, Reichel E. Transpupillary thermotherapy for age-related macular degeneration: long-pulse photocoagulation, apoptosis and heat shock proteins. Ophthalmic Surg Lasers 2000;31:359-73.

9. Reichel E, Berrocal AM, Ip M, et al. Transpupillary thermotherapy of occult subfoveal choroidal neovascularisation in patients with age-related macular degeneration. Ophthalmology 1999;106:1908-14.

10. Odergren A, Algvere PV, Seregard S, et al. A prospective study on transpupillary thermotherapy (TTT) versus photodynamic therapy (PDT) for neovascular age-related macular degeneration. Br J Ophthalmol 2008;92:757-61.

11. Ming Y, Algvere PV, Odergren A, et al. Subthreshold transpupillary thermotherapy reduces experimental choroidal neovascularisation in the mouse without collateral damage to the neural retina. Invest Ophthalmol Vis Sci 2004;45:1969-74.

12. Odergren A, Ming $Y$, Kvanta A. Photodynamic therapy of experimental choroidal neovascularization in the mouse. Curr Eye Res 2006;31:765-74.

13. Costa RA, Jorge R, Calucci $D$, et al. Intravitreal bevacizumab (Avastin) in combination with verteporfin photodynamic therapy for choroidal neovascularization associated with age-related macular degeneration (IBeVe Study). Graefes Arch Clin Exp Ophthalmol 2007;245:1273-80.

14. Kumar A, Gopalakrishnan K, Sinha S. Combination photodynamic therapy and intravitreal ranibizumab in neovascular $\mathrm{AMD}$ in a north Indian population. Retina 2008;28:1132-7.

15. Lazic R, Gabric N. Verteporfin therapy and intravitreal bevacizumab combined and alone in choroidal neovascularization due to age-related macular degeneration. Ophthalmology 2007;114:1179-85

16. Vallance $\mathbf{J H}$, Johnson B, Majid MA et al. A randomised prospective double-masked exploratory study comparing combination photodynamic treatment and intravitreal ranibizumab vs intravitreal ranibizumab monotherapy in the treatment of neovascular age-related macular degeneration. Eye (Lond) 2010;24:1561-7.

17. Odergren A, Algvere PV, Seregard S, et al. Vision-related function after low-dose transpupillary thermotherapy versus photodynamic therapy for neovascular agerelated macular degeneration. Acta Ophthalmol 2010;88:426-30.

18. Lalwani GA, Rosenfeld PJ, Fung AE, et al. A variable-dosing regimen with intravitreal ranibizumab for neovascular age-related macular degeneration: year 2 of the PrONTO study. Am J Ophthalmol 2009;148:43-58.

19. Holz FG, Amuako W, Donate J, et al. Safety and efficacy of a flexible dosing regimen of ranibizumab in neovascular age-related macular degeneration: the SUSTAIN Study. Ophthalmology 2011;118:663-71. 